\section{Case Reports in Ophthalmology}

\title{
Tamoxifen Use in a Patient with Idiopathic Macular Telangiectasia Type 2
}

\author{
Alice Behrens Ahmed Sallam John Pemberton Sami Uwaydat \\ Jones Eye Institute, University of Arkansas for Medical Sciences, Little Rock, AR, USA
}

\section{Keywords}

Tamoxifen · Macular telangiectasia · Fluorescein angiography · Optical coherence tomography angiography

\begin{abstract}
Crystalline deposits and neurosensory retinal cavitary changes can develop in both tamoxifen retinopathy and nonproliferative idiopathic macular telangiectasia type 2 (MacTel2). MacTel2 is typically differentiated from tamoxifen retinopathy based on the presence of late leakage and mid-phase telangiectatic vessels on fluorescein angiography (FA) and the presence of hyperautofluorescence. Unlike MacTel2, tamoxifen retinopathy is known to be a progressive disease and the cessation of tamoxifen results in resolution of retinopathy. We report a unique case of nonproliferative MacTel2 in a 36-year-old Hispanic woman with tamoxifen use and the vision outcome 30 months after cessation of tamoxifen. The FA and optical coherence tomography angiography findings of this patient support the diagnosis of MacTel2, but her cessation of tamoxifen led to partial reversal of the topographic findings and improvement in visual acuity. This patient is also unique in the unusually young age of presentation for MacTel2. Our case supports that there are common pathways in the pathogenesis of tamoxifen retinopathy and MacTel2, and tamoxifen use could potentially accelerate foveal atrophy in patients with MacTel2.




\section{Introduction}

Tamoxifen retinopathy usually manifests as crystalline maculopathy with or without cystoid macular edema (CME) [1,2]. With the introduction of optical coherence tomography (OCT), structural changes in the fovea evidenced by loss of outer retinal layers or cavitation of inner retinal layers or outer retinal layers have been described [3-7]. Clinical features of nonproliferative macular telangiectasia type 2 (MacTel2) include reduced retinal transparency, crystalline deposits, ectatic capillaries, blunted venules, retinal pigment plaques, and foveal atrophy. A characteristic angiographic pattern of MacTel2 is late-phase leakage of telangiectatic macular capillaries. Neurosensory atrophy on OCT, crystalline deposits, and neurosensory retinal cavitary changes can develop in both tamoxifen retinopathy and MacTel2. Fluorescein angiography (FA) and optical coherence tomography angiography (OCTA) are helpful to differentiate between the 2 conditions.

We present a young patient with MacTel2 with advanced foveal atrophy after a low accumulative dose of tamoxifen.

\section{Case Presentation}

Our patient is a 36-year-old Hispanic female who presented with an 8-month history of gradual vision decline in both eyes. She had been taking $20 \mathrm{mg}$ of tamoxifen daily for 33 months (cumulative dose $20.7 \mathrm{~g}$ ) as chemotherapy for a previously excised estrogen receptor-positive, grade II invasive ductal carcinoma. Her best-corrected vision was 20/60 in the right eye and 20/200 in the left eye. Eye exams were significant for a blunt foveal reflex with parafoveal retinal opacification and fine yellow intraretinal nonrefractile deposits (Fig. 1a, d). Fundus autofluorescence showed absence of the normal foveal hypoautofluorescence (Fig. 1b, e). FA revealed diffuse deep perifoveal late leakage in both eyes (Fig. 1c, f). Spectral domain high-definition (HD) OCT of the right eye revealed a large outer foveal cyst with disrupted retinal pigment epithelium and outer retinal bands, and thinning of the foveal layers (Fig. 2a). In the left eye, OCT examination showed a defect in the cone outer segments and ellipsoid zone of the fovea (Fig. 2c). There was no CME. After tamoxifen was stopped, anastrozole $1 \mathrm{mg}$ daily was started. Thirty months later, her best-corrected vision was 20/50 in the right eye and 20/70 in the left eye. Macular HD OCT showed a decrease in the size of the foveal cavitation in the right eye (Fig. 2b) and partial resolution of the defects of the ellipsoid zone and the cone outer segments of the left eye (Fig. 2d). Angioplex OCTA on the most recent office visit of the fovea in both eyes revealed thinning of the capillary plexus with a coarse branching pattern in the inner and middle retinal layers (Fig. 3a, b, d, e), with vascular invasion of the normally avascular outer retinal layers (Fig. 3c, f), more prominent in the right eye. These OCTA findings are consistent with the diagnosis of MacTel2, as described by Spaide et al. [7].

\section{Discussion}

Tamoxifen is currently the only FDA-approved selective estrogen-receptor-modulator adjuvant therapy for early-stage breast cancer with positive estrogen receptor [8]. The current therapeutic dosage starts at $20 \mathrm{mg}$ daily and increases up to $40 \mathrm{mg}$ daily. Ocular toxicity includes keratopathy, cataract, and optic neuritis, but most commonly presents as a crystal- 
line retinopathy with or without CME $[1,2]$. Tamoxifen usage has also been implicated in the formation of macular holes $[9,10]$. Ocular findings are generally observed after a cumulative dose of $100 \mathrm{~g}$ or more or a daily use of $120 \mathrm{mg}$ or more [6]. With the advent of OCT, several reports described the formation of cystic cavitary spaces in the retina, even in patients with low cumulative dosage $[5,6]$.

Tamoxifen can cause retinal damage through multiple mechanisms. Being cationic and amphophilic, tamoxifen can cause drug-polar lipid complexes accumulating in lysosomes, inducing cell oxidative damage. Müller glia is essential in maintaining retinal tissue integrity and hemostasis. Tamoxifen inhibits the glutamate-aspartate transporter in Müller cells, leading to excessive intracellular accumulation of glutamate, Müller cell dysfunction and apoptosis, vascular remodeling, and neurodegeneration of the retinal layers [11, 12].

While it is possible that the retinal damage in our patient was caused by tamoxifen use, there are several points, which support that this patient had pre-existing MacTel2. First, most patients with tamoxifen-related pseudocystic changes do not exhibit macular leakage on FA [13]. Our case had significant fluorescein leakage in the absence of CME, a feature classically observed in MacTel2 and the pattern of temporal and nasal leakage was consistent with Class 2 FA findings of MacTel2 [13]. Second, the presence of significant capillary thinning and remodeling of the superficial and deep retinal capillaries on OCTA further support the presence of retinal telangiectatic changes, which is consistent with the diagnosis of MacTel2 [8]. MacTel2 usually manifests at the sixth and seventh decades, and only about 3\% of MacTel2 first manifests before the age of 40 years [14]. The young age and the advanced foveal changes of this patient suggest that the natural progression of MacTel2 is unlikely to be the sole cause of her vision decline. In this case, it is conceivable that tamoxifen usage in the setting of pre-existing MacTel2 accelerated the foveal atrophy, even with a relatively low total dose of tamoxifen. The decrease in the cavitary spaces noted in our patient may also be due to hypertrophy of adjacent cells, recovery of damaged cells, or collapse of retinal cyst walls.

Thirty months after discontinuing tamoxifen, the vision in our patient improved slightly. Tamoxifen maculopathy, unlike MacTel2, appears to be a nonprogressive disease. Although Müller cells are not known to regenerate [12], it is possible that cessation of tamoxifen, once maculopathy is detected, can limit further macular damage and stabilize the vision loss.

\section{Conclusions}

Tamoxifen should be avoided in patients with suspected MacTel2. A baseline macular HD OCT and OCTA, along with a dilated eye exam, are recommended for all patients with MacTel2 prior to initiation of tamoxifen therapy.

\section{Statement of Ethics}

The authors have no ethical conflicts to disclose. The patient has given written consent for publication. 


\section{Disclosure Statement}

None of the authors has any conflict of interest related to the publication of this paper.

\section{Funding Sources}

This work was supported in part by Research to Prevent Blindness and an endowment from the Martha Wood Bentley Chair in Ophthalmology.

\section{References}

1 McKeown GA, Swartz M, Blom J, Maggiano JM: Tamoxifen retinopathy. Br J Ophthalmol 1981;65:177179.

2 Pavlidis NA, Petris C, Briassoulis E, Klouvas G, Psilas C, Rempapis J, Petroutsos G: Clear evidence that long-term, low-dose tamoxifen treatment can induce ocular toxicity. A prospective study of 63 patients. Cancer 1992;69:2961-2964.

-3 Gualino V, Cohen SY, Delyfer MN, Sahel JA, Gaudric A: Optical coherence tomography findings in tamoxifen retinopathy. Am J Ophthalmol 2005;140:757-758.

4 Georgalas I, Paraskevopoulos T, Papaconstaninou D, Brouzas D, Koutsandrea C: Large bilateral foveal cysts in the inner retina of a patient treated with tamoxifen, diagnosed with Fourier-domain optical coherence tomography. Clin Ophthalmol 2013;7:707-709.

5 Chung H, Kim D, Ahn SH, Kim JG, Lee JY, Lim JY, Yoon Y H: Early detection of tamoxifen-induced maculopathy in patients with low cumulative doses of tamoxifen. Ophthalmic Surg Lasers Imaging 2010;3:1-5.

-6 Doshi RR, Fortun JA, Kim BT, Dubovy SR, and Philip J, Rosenfeld PJ: Pseudocystic foveal cavitation in tamoxifen retinopathy. Am J Ophthalmol 2014;157:1291-1298.

7 Spaide, RF, Klancnik JM, Cooney MJ: Retinal vascular layers in macular telangiectasia type 2 imaged by optical coherence tomographic angiography. JAMA Ophthalmol 2015;133:66-73.

$>8$ Eisner A, Luoh S: Breast cancer medications and vision: effect of treatments for early-stage disease. Current Eye Research 2011;360:867-885.

-9 Cronin BG, Lekich CK, Bourke RD: Tamoxifen therapy conveys increased risk of developing a macular hole. Int Ophthalmol 2005;26:101-105.

10 Chung SE, Kim SW, Chung HW, Kang SW: Estrogen antagonist and development of macular hole. Korean J Ophthalmol 2010;24:306-309.

11 Maenpaa H, Mannerstrom M, Toimela T, Salminen L, Saransaari P, Tahti H: Glutamate uptake is inhibited by tamoxifen and toremifene in cultured retinal pigment epithelial cells. Pharmacol Toxicol 2002;91:116-122.

12 Goldman D: Müller glial cell reprogramming and retina regeneration: Nat Rev Neurosci 2014;15:431442.

13 Sallo FB, Leung I, Clemons TE, Peto T, Bird AC, Pauleikhoff D: Multimodal imaging in type 2 idiopathic macular telangiectasia. Retina 2015;35:742-749.

14 Heeren TF, Holz FG, Charbel Issa P: First symptoms and their age of onset in macular telangiectasia type 2. Retina 2013;34:916-919. 


\section{Case Reports in Ophthalmology}
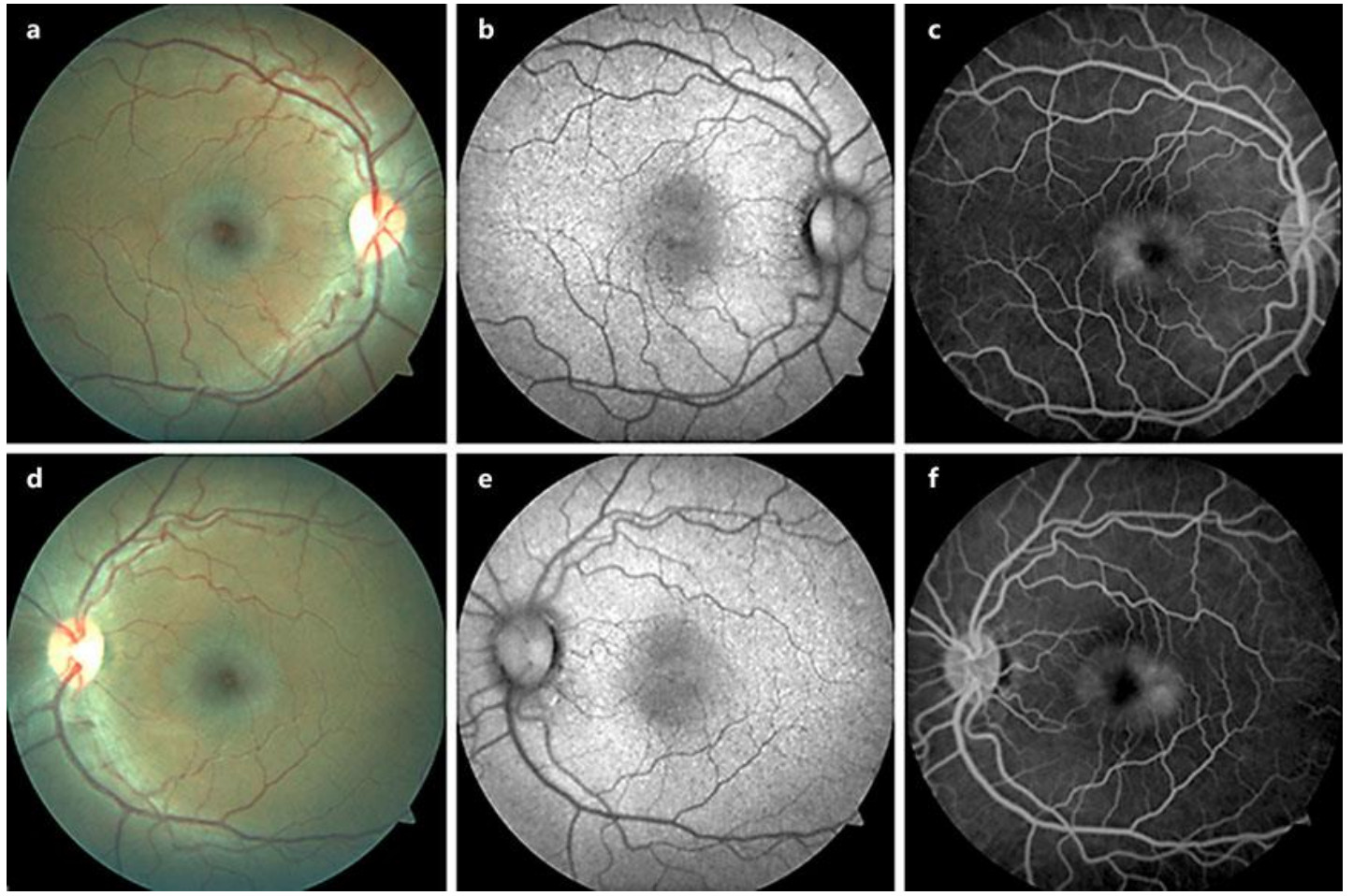

Fig. 1. Fundus photos of the initial visit of the right eye (a) and left eye (d). Fundus autofluorescence of the initial visit of the right eye (b) and left eye (e) showed absence of the normal foveal hypoautofluorescence. Late-phase fluorescein angiography of the initial visit of the right eye (c) and left eye (f) showed diffuse leakage. 


\section{Case Reports in Ophthalmology}

C 2018 The Author(s). Published by S. Karger AG, Basel www.karger.com/cop

Behrens et al.: Tamoxifen Use in a Patient with Idiopathic Macular Telangiectasia Type 2
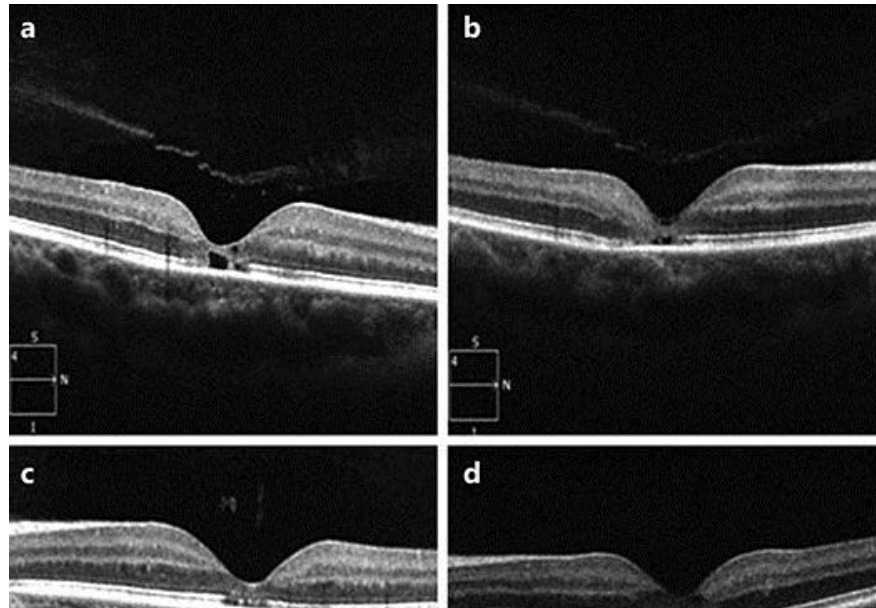

d

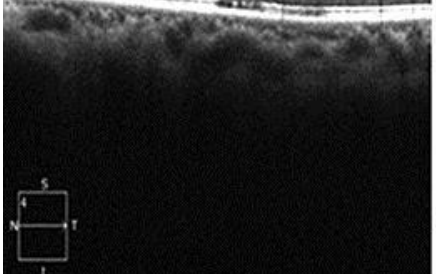

Fig. 2. Horizontal sections of optical coherence tomography on the initial visit showed a large foveal cyst in the right eye (a) and a defect of the cone outer segment and ellipsoid zone in the left eye (c). Horizontal section of optical coherence tomography on follow-up showed a decrease in the size of cavitation due to "collapse" of the roof in the right eye (b) and partial resolution of the initial defects in the ellipsoid zone and outer segments of the photoreceptors of the left eye (d). 


\section{Case Reports in Ophthalmology}

Case Rep Ophthalmol 2018;9:54-60 DOI: $10.1159 / 000485739$ (c) 2018 www.karger.com/cop

Behrens et al.: Tamoxifen Use in a Patient with Idiopathic Macular Telangiectasia Type 2
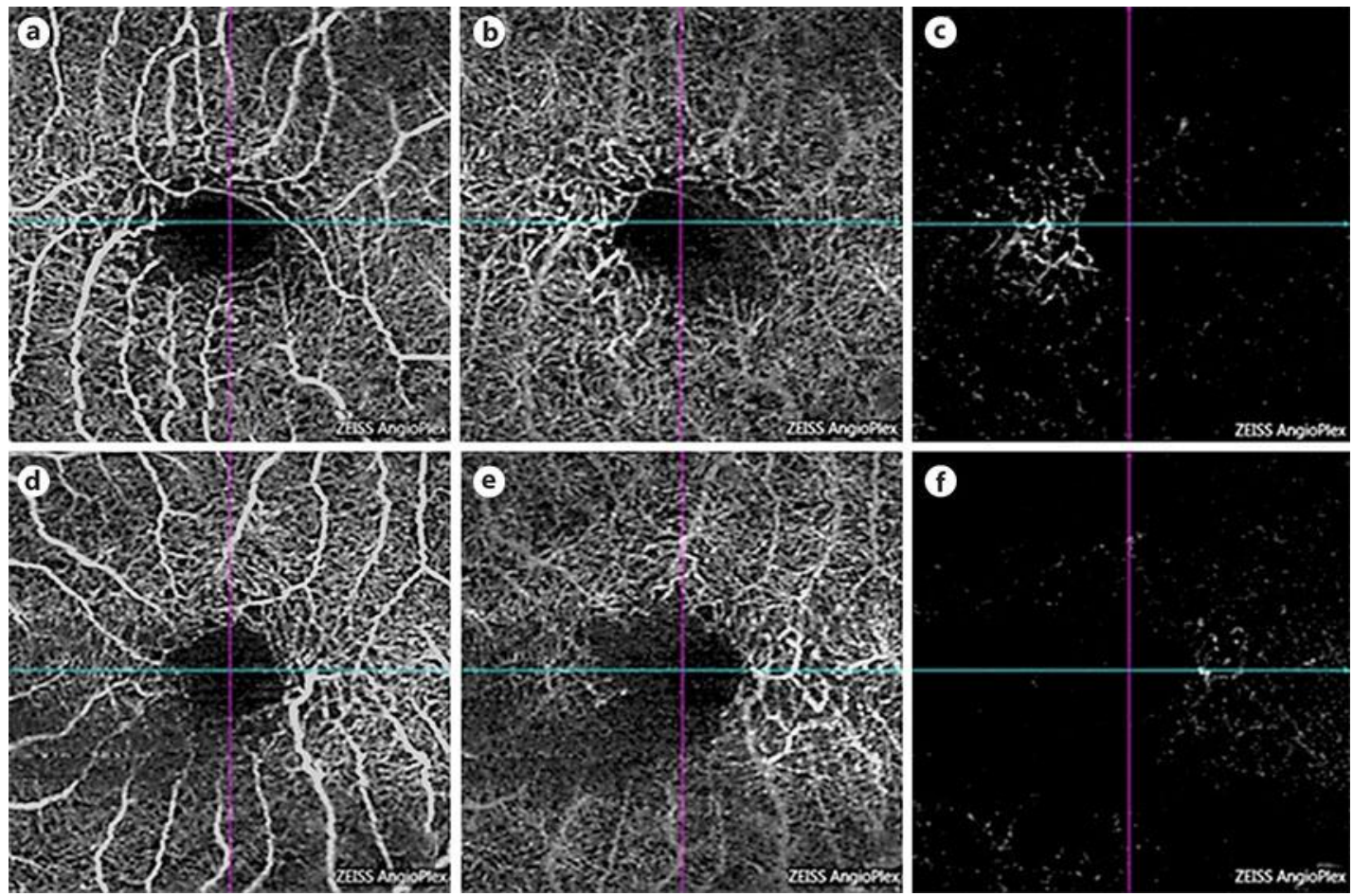

Fig. 3. Optical coherence tomography angiogram showed vascular remodeling and capillary plexus thinning in the inner retinal layers (right eye, a; left eye, d) and middle retinal layers (right eye, b; left eye, e) temporal to the fovea. Retinal vessels are noted in the normally avascular outer retina (right eye, c; left eye, f). 